\title{
RDA: A Real-Life Look
}

\author{
Megan Chellew \\ McGill University
}

For the last few years, every time someone said "RDA" to me, it conjured up a vision of a very complicated chart of FRBR (Functional Requirements for Bibliographic Records) relationships, swirling around with new rules and weird-looking bibliographic records. I love rules, and the chart of FRBR relationships is a really exciting way to look at things. But I need concrete examples to really understand something new; for me, the burning question was: "What is this really going to look like?"

On October 1, 2010, the United States testing period began for RDA (Resource Description and Access), the new cataloguing standard. Since then, RDA records have been appearing in OCLC, available for export and for the world to see. For the first time, we can look at the reality of RDA, from both the public view and the cataloguer's view. I've chosen to highlight the changes that will affect virtually each and every record; there are additional changes that will either not be seen frequently, or will be implemented at the cataloguer's discretion. It's also important to keep in mind that the public view is something that will be customized by each individual library. Some institutions may choose not to display some of the new RDA features. The examples I've chosen are from institutions that are choosing to display these features.

Looking at a record from the Library of Congress catalogue (fig. 1), one feature immediately jumps out and gets right to the heart of the changes RDA has brought to cataloguing. At the bottom of the record are three new fields, labeled as Content Type, Media Type and Carrier Type. These fields use controlled vocabularies to indicate what the resource really is. It is a score, so the content type is notated music. No extra media is needed to access the resource, so the media type is unmediated. And the carrier type, the actual physical reality of the resource, is a volume. (All the RDA controlled vocabularies can be found at: http://metadataregistry.org/rdabrowse.htm.) With the exception of these three new fields, this bibliographic record looks very similar to the AACR2 records we're used to seeing. From the public service point of view, the changes RDA has brought are very straightforward and accessible.

(C) 2010 The author and the Canadian Association of Music Libraries, Archives and Documentation Centres / L'auteur et l'Association canadienne des bibliothèques, archives et centres de documentation musicaux 


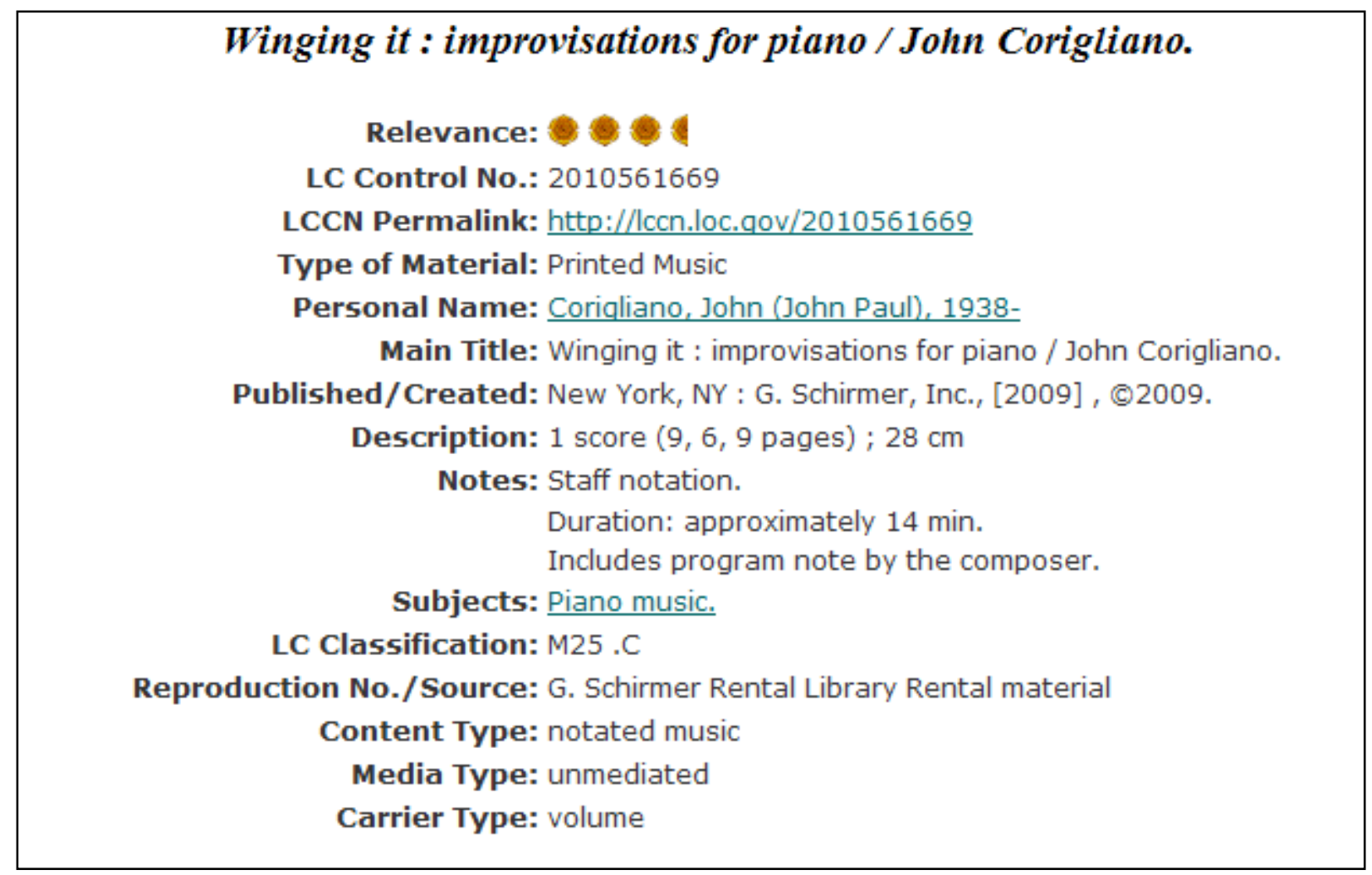

Fig. 1

Looking at the MARC view of the same record (fig. 2), cataloguers will want to know what will tell them that this is an RDA record. First of all, in the 040 field, subfield e, "rda" is given as the Convention of Description. The second clue is in the Leader (the 000 field in this record). The Descriptive Cataloging Form (position 18), which we are used to seeing coded as "a" for AACR2 records, in RDA is given as " $i$ " because it is non-AARC2. Further down the record, cataloguers will find the three new fields mentioned above: 336 (Content Type), 337 (Media Type) and 338 (Carrier Type). Each of these fields has a subfield 2, indicating which controlled vocabulary is used to fill in the terms in the subfield a. The subfield 2 (rdacontent, rdamedia, rdacarrier) is a further clue that this is an RDA record.

There are a few other differences that RDA brings to this record, none of which diverge wildly from what we're used to seeing. The 260 field (Production, Distribution, etc.), subfield c, has two identical dates recorded-[2009], (C2009-which does look unusual. The RDA rule is to record both the copyright date and the date of publication, even if they are the same year. How these dates are transferred to the corresponding positions of the 008 field is subject to interpretation. In this record, they are recorded as " $t$ " (publication date and copyright date) with 2009 entered in both date positions. Other institutions are recording this as "s" (single date) when the publication and copyright dates are identical, and only entering 2009 in the first date position. 
Another small difference is in the 300 field (Physical Description). This piece of music is for solo piano, and according to AACR2 rules, would have been designated as "pages of music" and not a "score" (score being reserved for music for more than one instrument or voice). Under RDA, however, this terminology has been normalized, and all notated music is referred to as a score.

\begin{tabular}{|c|}
\hline 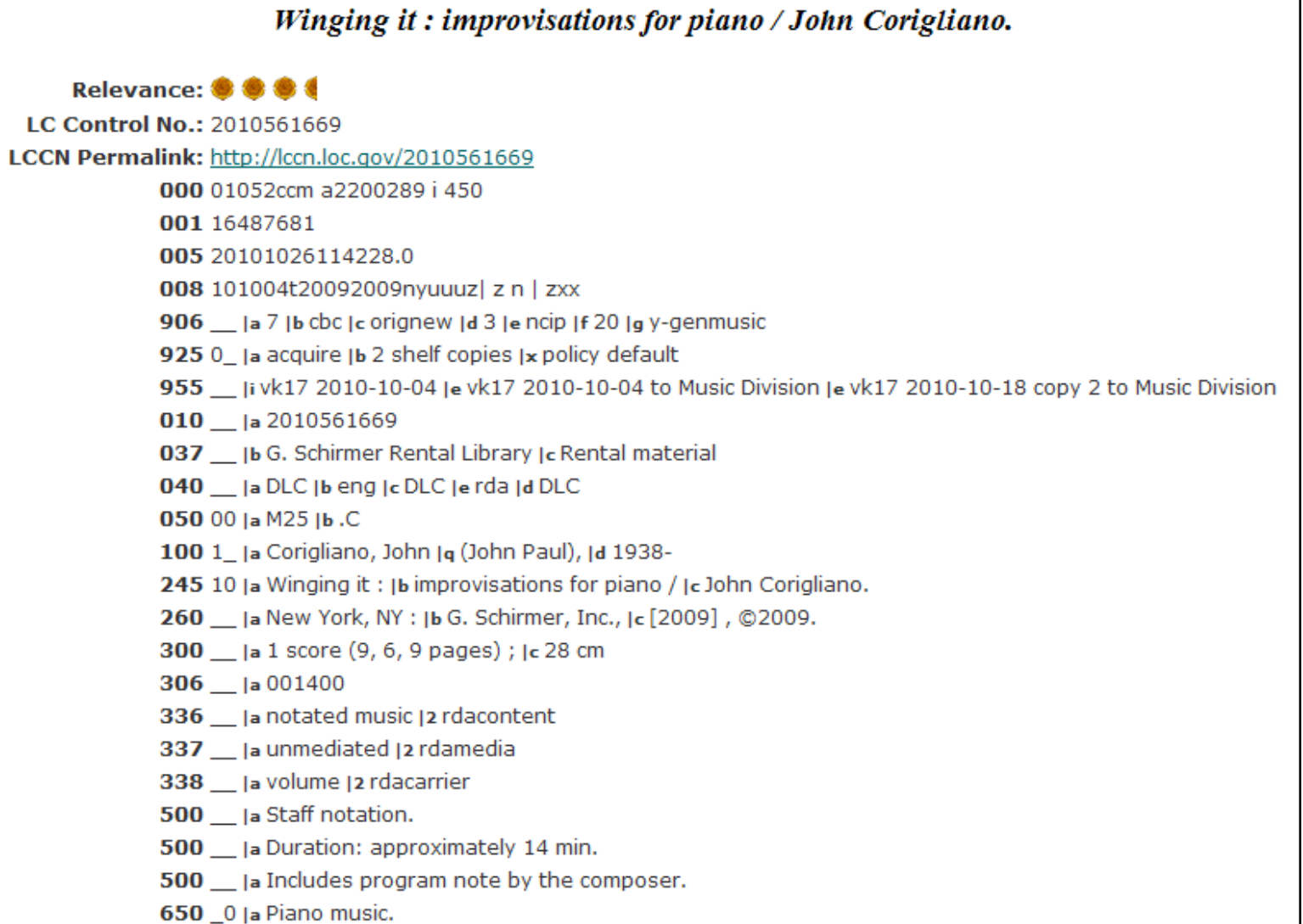 \\
\hline
\end{tabular}

Fig. 2

In several places, you will see words that would have been abbreviated in the past written out in full. RDA has abolished the use of the abbreviations (unless an abbreviated form is what appears on the resource in hand). So, instead of "p.", we have "pages", and instead of "approx." (in the 500 field indicating the duration of the piece), we see "approximately". The exception to this rule is that the physical dimensions of the score are still recorded in "cm", not centimetres. In RDA, "cm" is considered a symbol, not an abbreviation.

One last difference in this record, which probably would not be noticed by anyone who was unaccustomed to controlled vocabularies and authorized forms of name, is the form of name in the 100 field. The authorized form of Corigliano's name is actually Corigliano, John, 1938-. 
Library of Congress' policy, however, is to always include the full form of name, which is why what we see in the 100 field here is Corigliano, John (John Paul), 1938-. (This RDA form is added to the existing authority record for Corigliano in a 700 field.)

RDA has a slightly higher impact on bibliographic records for sound recordings. In the sound recording record in figure 3, again, we see the three new RDA fields. In the first example, we saw that Library of Congress has chosen to label them "Content Type", "Media Type", "Carrier Type". Here, the University of Chicago has labeled theirs "Content type", "Medium", "Format". In this case, the content is performed music. The resource requires Audio media to access it, which is recorded in the Medium field. And finally, the Format, the thing the music is recorded on, is an audio disc.

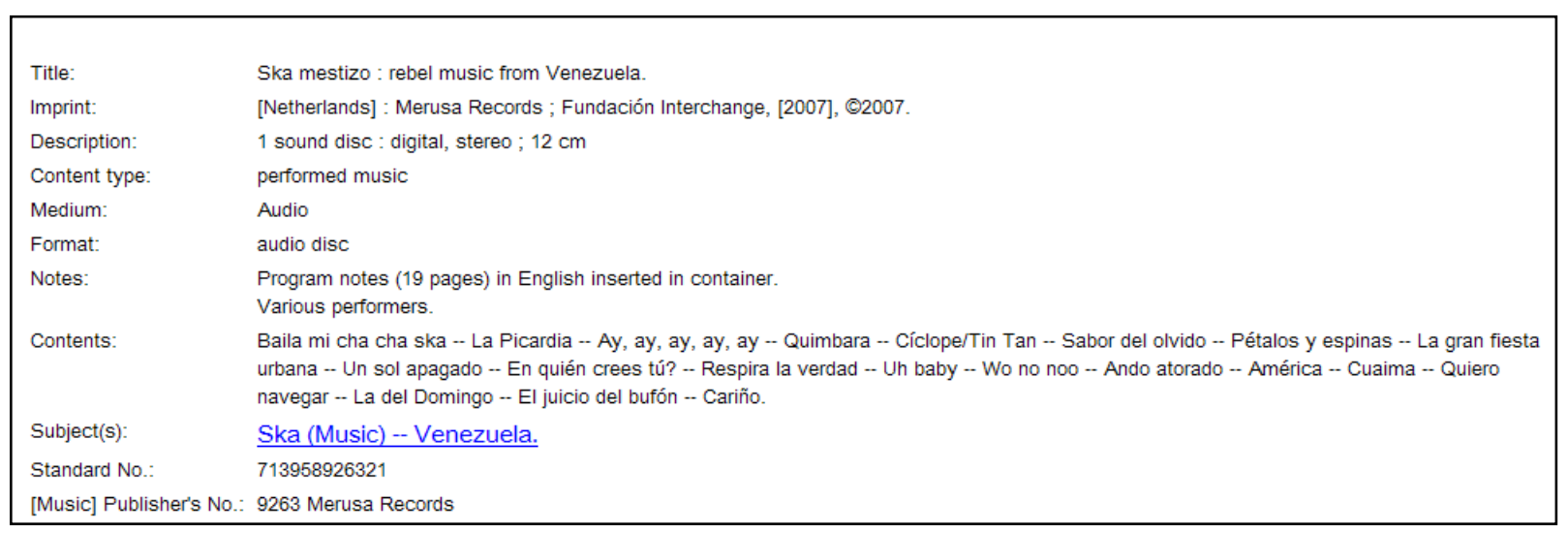

Fig. 3

Also, experienced users might notice that the Title field looks a little bare; the [sound recording] (also known as the GMD, or general material description), which would normally appear after the first part of the title, will no longer be used in RDA. That GMD is really what the three new fields replace. Those fields parse the information out more specifically, so it is more computer friendly.

Looking at the MARC view (fig. 4), a cataloguer will immediately notice that the 245 is missing its subfield $\mathrm{h}$ [sound recording] GMD. That change, along with the new 336, 337, 338 fields, is the major change in cataloguing rules for sound recordings. Again, there are minor changes, such as the subfield $\mathrm{c}$ in the 260 field, where the date of publication and copyright date are both recorded. Looking at the 008 field, this record is an example of an institution that has decided to code the date as a single date, since the date of publication and copyright are identical. Another minor change is in the 300 field, subfield b, where stereo no longer has a period at the end. It is no longer considered an abbreviation for stereophonic, but a word in its own right (mono gets the same treatment in that it is no longer considered an abbreviation for monaural). Also in the 300 
field, this time in subfield c, the dimensions of the disc are recorded in centimetres instead of inches. Because RDA is meant to be a truly international standard, physical dimensions will be recorded using the metric system, since most countries use the metric, and not the imperial, system.

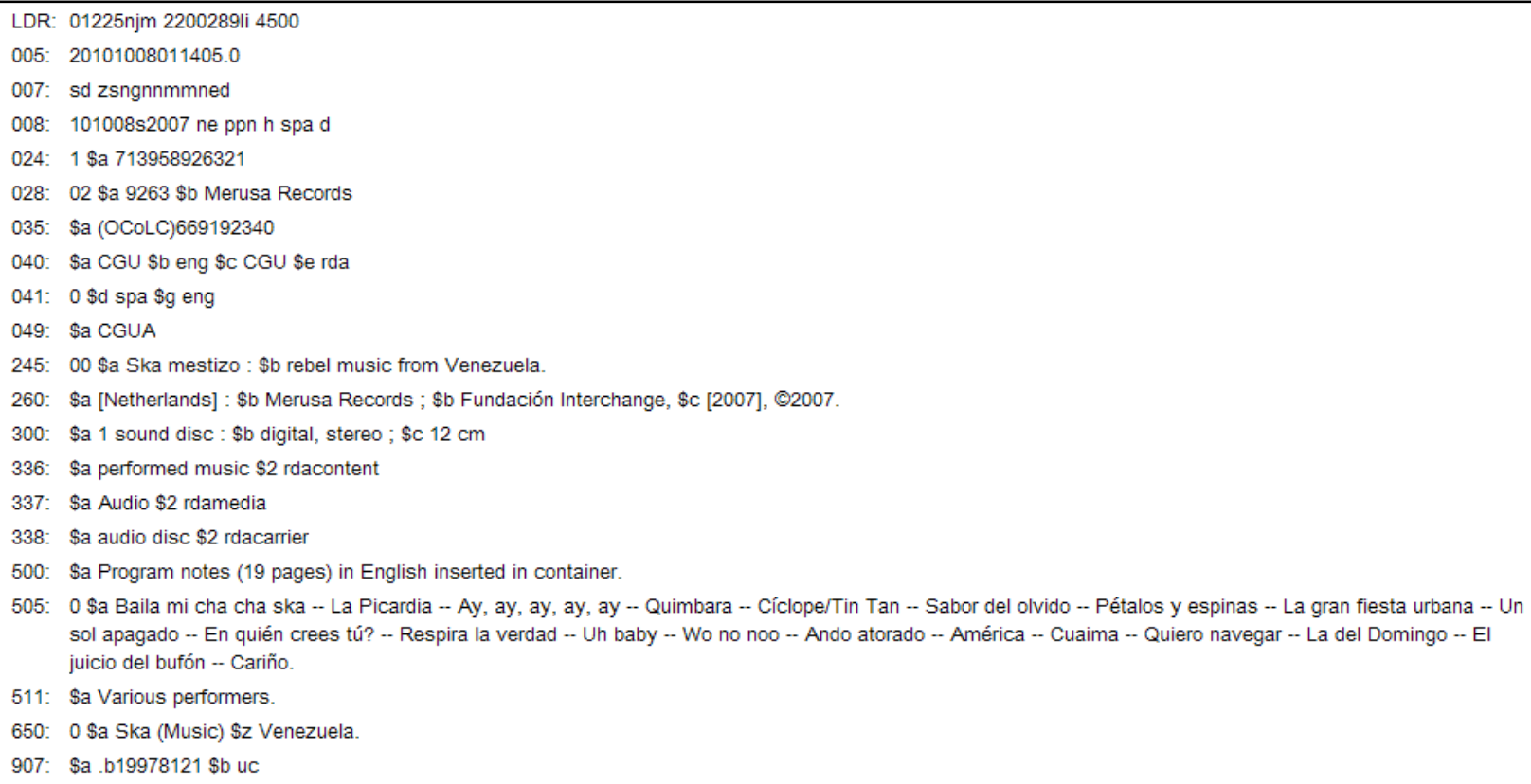

Fig. 4

One great strength of RDA is that it really highlights the relationships between the resource and the person, or people, involved in producing it. So, in the example shown in figure 5, in the Personal Author field, Satie is designated as the composer (using a subfield e and a relator term in the 100 field). In the second Added author field, Debussy's relationship is spelled out; he is the "arranger of music". By making the relationships clear, the user can easily see what each person's involvement was, without having to refer to different fields, and possibly put the puzzle pieces together themselves. In this example, it is easy to understand Debussy's role; it is clearly outlined in the Title (or 245) field. But there are certainly times where a user would have to look in a notes field to find out what Debussy's involvement was. It also means that resources on which Satie was the composer will index separately from resources on which he was, for example, the performer. This makes retrieval easier for both the computer and the searcher. Simply as a point of interest, it is worth noting in this record, that the uniform title is not, in fact, constructed according to any RDA rules. It is, however, a very interesting idea to include the instrumentation the piece was arranged for, and the arranger. 


\section{Gymnopédies II : harpe}

Satie, Erik (Erik Alfred Leslie), 1866-1925, composer.

Personal Author: Satie, Erik (Erik Alfred Leslie), 1866-1925, composer.

Uniform title: Gymnopédies. No 2. arranged: orchestra (Debussy)

Title: Gymnopédies II : harpe / de Erik Satie ; orchestrées par Claude Debussy.

Publication info: Boca Raton, Florida : Edwin F. Kalmus \& Co., Inc., [198-?]

Physical description: 2 parts ; $33 \mathrm{~cm}$

Content type: notated music

Medium: unmediated

Format: volume

General Note: Harp parts only, for orchestral arrangement; originally for piano solo.

General Note: Caption title.

Local note: International Harp Archives.

Local note: Zingel, Orchestra studies.

Subject term: Harp--Orchestral excerpts.

Subject term: Orchestral music, Arranged--Parts.

Added author: Satie, Erik (Erik Alfred Leslie), 1866-1925. Gymnopédies. No 2

(work).

Added author: Debussy, Claude (Achille Claude), 1862-1918, arranger of music.

Publisher's no.: A 1991 Edwin F. Kalmus \& Co., Inc.

Collection note: Gift of:Victor Salvi Foundation.

Fig. 5

Anyone wanting to look at further examples of RDA records can pull a set from OCLC (either the browser or the client) by doing a command search by entering the following: dx:rda, and then adding another term (for example, su:music or au:Debussy) to refine the search and retrieve a manageable set of records.

None of the changes that I've shown in the above records is really a great departure from the AACR2 rules we have come to know and love. Cataloguers are still recording the same kinds of information that we always have. With RDA we'll be recording some information a little differently, or in a new location. But, fundamentally, these changes are all very manageable and understandable. This is, of course, a transition period. There will be further changes as RDA is implemented more fully. But the whole point of having a transition is to try to make the move from the AACR2 world to the new RDA world as smooth as possible. 